\title{
A study of the $\beta$ Cephei star $\gamma$ Pegasi: binarity, magnetic field, rotation, and pulsations ${ }^{\star}$
}

\author{
V. V. Butkovskaya ${ }^{1}$ and S. I. Plachinda ${ }^{1,2}$ \\ ${ }^{1}$ Crimean Astrophysical Observatory, Nauchny, Crimea, 98409, Ukraine \\ e-mail: varya@crao.crimea.ua \\ 2 Institute for Astronomy, University of Vienna, Tuerkenschanzstrasse 17, 1180 Vienna, Austria
}

Received 6 May 2006 / Accepted 2 April 2007

ABSTRACT

\begin{abstract}
Aims. We provide observational material to study the magnetic field variability of the classical $\beta$ Cep-type star $\gamma$ Peg. Methods. The observations were carried out in the He I 6678 line in the course of 23 observing nights from 1997 to 2005 with using the Coudé spectrograph in spectropolarimetric mode at the Crimean $2.6 \mathrm{~m}$ telescope. The behavior of stellar wind was studied in the UV region using data from the IUE satellite (the INES database).

Results. It is shown that the UV stellar wind exhibits a variability. A variation of the wind due to stellar pulsations has been detected. In the He I 6678 line, the abnormally blueshifted radial velocities $\left(\gamma=-60.57 \pm 0.29 \mathrm{~km} \mathrm{~s}^{-1}\right)$ were detected during a single night in 2005. We do not confirm the 370.5-day orbital period. The most probable orbital period was estimated as $P_{\text {orb }}=6.81608 \pm 0.00012$ day. The ratio $P_{\text {orb }} / P_{\text {puls }}=44.92$ appeared to be very close to integer. We have detected the presence of a weak magnetic field on the star. The longitudinal component of the field varies from $-10 \mathrm{G}$ to $30 \mathrm{G}$ with the stellar rotation. The most probable rotational period is $P_{\text {rot }}=6.6538 \pm 0.0016$ days. Both the orbital and the rotational periods are integral multiples of the difference between them: $P_{\text {orb }} /\left|P_{\text {orb }}-P_{\text {rot }}\right|=42.002$, and $P_{\text {rot }} /\left|P_{\text {orb }}-P_{\text {rot }}\right|=41.002$. Variation in the longitudinal magnetic field during the pulsation period with an amplitude about $7 \mathrm{G}$ was detected.
\end{abstract}

Key words. stars: magnetic fields - stars: early-type - stars: oscillations - stars: binaries: spectroscopic - stars: individual: $\gamma$ Pegasi

\section{Introduction}

The classical $\beta$ Cep-type star $\gamma$ Peg (HD 886, HR 39, Sp B2 IV) exhibits low-order purely radial pulsations. It has one of the weakest amplitude variations in radial velocity $2 K=7 \mathrm{~km} \mathrm{~s}^{-1}$, light $\Delta m_{\mathrm{v}}=0.017$ with short pulsation period of 0.15 day, and de Jager et al. (1982) concluded that $\gamma$ Peg has a virtually zero rotational velocity component; i.e., the star is seen rotation pole-on.

McNamara (1955) pointed out a possibility that the $\gamma$-axis of the 0.15-day velocity curve of $\gamma$ Peg varies. Harmanec et al. (1979) determined the 6.83-day period for the variations in the $\gamma$-axis and concluded that the star is a spectroscopic binary with a circular, slightly inclined orbit. Ducatel et al. (1981) also detected the day-to-day variations of the $\gamma$-axis, but they suggest that these variations are associated with stellar oscillations.

According to analysis of high-resolution observations of this star that were carried out in a period from 1997 to 2005, Butkovskaya et al. (2006) concluded that $\gamma$ Peg is a spectroscopic binary as suggested by Harmanec et al. (1979). Recently, Chapellier et al. (2006), combining their radial velocity measurements with data from the literature, confirmed the binarity of this star but with another orbital period of 370.5 days.

The first attempt to detect a magnetic field on $\gamma$ Peg was made by Babcock (1958). He found no evidence of a magnetic field in this sharp-line star. Rudy \& Kemp (1978) measured the

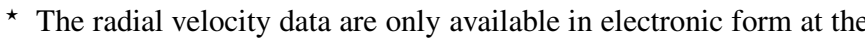
CDS via anonymous ftp to

cdsarc.u-strasbg.fr $(130.79 .128 .5)$ or via

http://cdsweb.u-strasbg.fr/cgi-bin/qcat?J/A+A/469/1069 circular polarization in the wings of the Balmer line $H_{\gamma}$ and detected no statistically significant magnetic field on the star with the least error $\sigma=30 \mathrm{G}$. However, using $\chi^{2}$ statistics, they concluded that $\gamma$ Peg shows strong evidence of a magnetic field. Despite this fact, the study presented by Landstreet (1982) showed no traces of a magnetic field on $\gamma$ Peg with the least error $\sigma=45$ G. Butkovskaya \& Plachinda (2004) also detected no statistically significant values of longitudinal magnetic field in this star. The detection level with error bars from 4 to $15 \mathrm{G}$ was achieved by them in the course of ten observing nights.

By now, direct magnetic field detections have been reported for a number of chemically normal, non-degenerated hot stars: $\beta$ Cep (B1 IV, Donati et al. 2001), $\theta^{1}$ Ori C (O4-6 V, Donati et al. 2002; Wade et al. 2006), V 2052 Oph (B2 IV-V), $\zeta$ Cas (B2 IV), and $\omega$ Ori (B2 IIIe) (Neiner et al. 2003a-c). The authors fit their data by means of an oblique magnetic dipole. These fits lead to polar field strengths of $360 \mathrm{G}$ for $\beta \mathrm{Cep}, 250 \mathrm{G}$ for V $2052 \mathrm{Oph}$, $335 \mathrm{G}$ for $\zeta$ Cas, $530 \mathrm{G}$ for $\omega$ Ori, and $1100 \mathrm{G}$ for $\theta^{1}$ Ori C.

In the extended magnetic survey presented by Hubrig et al. (2006), the longitudinal magnetic field of a few hundred Gauss was detected on the $\beta$ Cep star $\xi^{1}$ CMa and 13 SPB stars. Discovery of a magnetic field with an unusually complex topology on the young massive star $\tau$ Sco is reported by Donati et al. (2006).

The real surface geometry, as well as the origin of magnetic field in chemically normal massive stars, has not been understood well. In addition to the hypothesis about the fossil origin of stellar magnetism, various authors consider the possibility of the magnetic field generation by a dynamo action in the convective cores (Charbonneau \& MacGregor 2001), as well as 
in the non-convective interiors of massive stars (Spruit 2002; MacDonald \& Mullan 2004; Mullan \& MacDonald 2005).

Two hot pulsating stars that host magnetic field $\beta$ Cep and V 2052 Oph pulsate mainly in the radial mode. No results of a study of magnetic field modulation due to the pulsations has been published for these stars.

The presence and pulsational modulation of the magnetic field of cool pulsating supergiants RR Lyr and $\eta$ Aql have been widely discussed in the literature. Babcock (1958) reported detection of a strong longitudinal magnetic field in RR Lyr showing variations between $B_{\mathrm{e}}=-1580$ and $+540 \mathrm{G}$ with probable errors less than $220 \mathrm{G}$. Babcock did not find any correlation of the magnetic field behavior with the pulsation period. Preston (1967) failed to detect any magnetic field on RR Lyr with 50 photographic observations obtained over two years (1963 and 1964). Romanov et al. $(1987,1994)$ obtained 83 Zeeman spectrograms with the $6 \mathrm{~m}$ telescope of the Russian Special Astrophysical Observatory on several nights in 1978, 1982, 1983, and 1984 with errors between 100-500 G. They reported that the magnetic field varies with amplitude up to $1.5 \mathrm{kG}$ over the pulsation cycle $(0.567 \mathrm{~d})$, while its average intensity shows a periodic long-term variation corresponding to the $40.8 \mathrm{~d}$ Blazhko period. Chadid et al. (2004) reports a series of high-precision (median $\sigma_{\mathrm{B}} \sim 80 \mathrm{G}$ ) longitudinal magnetic field measurements of RR Lyr obtained over a period of almost 4 years from 1999-2002. These data provide no evidence of a strong magnetic field in the photosphere of RR Lyr. Plachinda (2000) reports the detection of a $\sim 100 \mathrm{G}$ longitudinal magnetic field in the photosphere of the classical Cepheid $\eta$ Aql with median $\sigma_{\mathrm{B}} \sim 37 \mathrm{G}$. The magnetic field shows periodic variations with a reversal of its polarity due to stellar pulsations $\left(-100<B_{\mathrm{e}}<+50 \mathrm{G}\right)$. Highprecision measurements of the longitudinal magnetic field of $\eta$ Aql (with errors between 3-5 G) using MuSiCoS polarimeter have shown no convincing evidence of a photospheric magnetic field (Wade et al. 2002). This result agrees with measurements by Borra et al. (1981). We continue spectropolarimetric monitoring of $\gamma$ Peg for high-precision measurements of the magnetic field. In this paper we present the results of this monitoring and study of magnetic field variations due to the stellar radial pulsations.

Smith \& McCall (1978) suggested that $\gamma$ Peg is a candidate for mild mass loss $\left(\sim 10^{-9} M_{\odot} \mathrm{yr}^{-1}\right)$. On the other hand, an investigation of the UV stellar wind provides very useful indirect information for the presence of a magnetic field in hot stars because the magnetic field specifically modulates the outflowing stellar wind (Neiner et al. 2003a-c). Therefore, in Sect. 2 we present the UV and spectropolarimetric observations. A search for UV stellar-wind variability is reported in Sect. 3 using data from the INES database. In Sect. 4 we tested the reality of the 370-day orbital period. The discovery of the presence of magnetic field on $\gamma$ Peg is reported in Sect. 5. Also, in Sect. 5 the results of a detail study of the longitudinal magnetic field variation due to the radial pulsations of the star are presented. All obtained results are summarized in Sect. 6.

\section{Observations and data reduction}

High-dispersion ultraviolet spectra were obtained with the Short Wavelength Prime (SWP) camera onboard the International Ultraviolet Explorer (IUE) satellite. Twenty-seven highdispersion UV spectra of $\gamma$ Peg were retrieved from the INES database. Table 1 presents the journal of the 25 spectra of $\gamma$ Peg obtained from 1978 to 1994 . Column 1 indicates the number of spectrum in the IUE archive. Column 2 gives the Heliocentric
Table 1. Journal of IUE observations of $\gamma$ Peg.

\begin{tabular}{cccccc}
\hline \hline $\begin{array}{c}\text { Image } \\
\text { swp }\end{array}$ & HJD & $\begin{array}{c}\text { Puls. } \\
\text { phase }\end{array}$ & $\begin{array}{c}\text { Image } \\
\text { swp }\end{array}$ & HJD & $\begin{array}{c}\text { Puls. } \\
\text { phase }\end{array}$ \\
\hline 03110 & 3804.82 & 0.063 & 05721 & 4060.15 & 0.613 \\
03946 & 3892.56 & 0.212 & 52770 & 9666.66 & 0.223 \\
05249 & 4010.18 & 0.290 & 52771 & 9666.69 & 0.395 \\
05250 & 4010.20 & 0.455 & 52772 & 9666.71 & 0.545 \\
05251 & 4010.23 & 0.615 & 52773 & 9666.73 & 0.692 \\
05252 & 4010.25 & 0.748 & 52774 & 9666.76 & 0.842 \\
05253 & 4010.27 & 0.884 & 52775 & 9666.78 & 0.994 \\
05254 & 4010.29 & 0.018 & 52776 & 9666.80 & 0.147 \\
05255 & 4010.31 & 0.152 & 52777 & 9666.83 & 0.300 \\
05256 & 4010.33 & 0.281 & 52783 & 9668.84 & 0.567 \\
05257 & 4010.35 & 0.437 & 52784 & 9668.86 & 0.717 \\
05258 & 4010.37 & 0.568 & & & \\
05259 & 4010.39 & 0.694 & & & \\
05260 & 4010.41 & 0.852 & & & \\
\hline
\end{tabular}

Julian Date at mid-exposure minus 2440000 . Column 3 indicates the pulsation period phases calculated using the ephemeris given in the Sect. 5.2. Two of the available spectra were discarded due to their poor quality. The spectra were mapped onto an equidistant wavelength with a grid spacing of $0.1 \AA$.

An intensive study of $\gamma$ Peg was performed in the He I 6678 line in the course of 23 nights from 1997 to 2005. In total 405 exposures were obtained using Coude spectrograph of the 2.6-m Shajn telescope of the Crimean Astrophysical Observatory (in particular, 262 exposures were obtained using polarimeter). Signal-to-noise ratios of a single spectrum were typically 350-600 with spectral resolving power of $2.2 \times 10^{4}$.

The study of the magnetic field of the star was carried out with the same equipment and "Flip-Flop" procedure that have already been discussed in detail by Plachinda \& Tarasova (1999), Plachinda (2004), and Plachinda (2005). We carried out the sequence of individual subexposures on the stellar object with the entrance achromatic quarter-wave plate rotated by $\pm 90^{\circ}$. See below the main mathematical conception of our data processing.

Let the entrance quarter-wave plate be offset by 90 degrees during the second exposure relative to its position during the first exposure. Then the displacement caused by splitting the atomic energy levels in the magnetic field of a star produces a wavelength shift $\Delta \lambda_{B}$, equal to

$\Delta \lambda_{B}=\left(e / 4 \pi m_{\mathrm{e}} c^{2}\right) z \lambda^{2} B_{\mathrm{e}} \cong 4.67 \times 10^{-13} z \lambda^{2} B_{\mathrm{e}}$,

where $e$ is the electronic charge, $m_{\mathrm{e}}$ its mass, $c$ velocity of light, $z$ the effective Lande factor, $\lambda$ the wavelength in $\AA$, and $B_{\mathrm{e}}$ the longitudinal magnetic field in Gauss.

Denote a center of gravity of a line with right circular polarization obtained during the first exposure by $\lambda_{1 r}$ and with left circular polarization by $\lambda_{1 l}$. The same designations for the successive exposures are: $\lambda_{2 r}$ and $\lambda_{2 l}$. Then, using Eq. (1), the mean longitudinal magnetic field is

$B_{\mathrm{e}}^{\prime}=k\left(\lambda_{1 r}-\lambda_{2 l}\right) / 2=k\left( \pm 2 \Delta \lambda_{B} \pm \Delta \lambda\right) / 2$

for the first pair of lines, where $\Delta \lambda_{B}$ is the displacement of the line caused by splitting of atomic energy levels in the magnetic field, $\Delta \lambda$ the displacement due to the instrumental effects, $k=$ $1 /\left(4.67 \times 10^{-13} z \lambda^{2}\right)$, and

$B_{\mathrm{e}}^{\prime \prime}=k\left(\lambda_{1 l}-\lambda_{2 r}\right) / 2=k\left(\mp 2 \Delta \lambda_{B} \pm \Delta \lambda\right) / 2$ 
for the other pair. Wavelength differences $\Delta \lambda_{B}$ and $\Delta \lambda$ are measured on successive exposures on the same part of the CCD. Subtracting (3) from (2), we obtain

$k\left( \pm 2 \Delta \lambda_{B} \pm \Delta \lambda\right) / 2-k\left(\mp 2 \Delta \lambda_{B} \pm \Delta \lambda\right) / 2=2 k \Delta \lambda_{B}$,

where the instrumental component of the shift, $\Delta \lambda$, cancels out.

To calculate values of displacements between centers of gravity $\lambda_{1 r}$ and $\lambda_{2 l}$, and also $\lambda_{1 l}$ and $\lambda_{2 r}$, we use the following formula:

$$
\begin{aligned}
2 \Delta \lambda_{B} \pm \Delta \lambda= & \left(\frac{\int \lambda\left(f^{*}(\lambda)-f(\lambda)\right) \mathrm{d} \lambda}{\int\left(f^{*}(\lambda)-f(\lambda)\right) \mathrm{d} \lambda}\right)_{1} \\
& -\left(\frac{\int \lambda\left(f^{*}(\lambda)-f(\lambda)\right) \mathrm{d} \lambda}{\int\left(f^{*}(\lambda)-f(\lambda)\right) \mathrm{d} \lambda}\right)_{2},
\end{aligned}
$$

where $\lambda$ is wavelength in $\AA, f^{*}(\lambda)$ the restriction level of a line profile under the continuum, and $f(\lambda)$ the function of a line profile (see Fig. 1 in Plachinda \& Tarasova 1999). The restriction level, $f^{*}(\lambda)$, is picked near the continuum in a point with an abrupt decline of a contour gradient value.

In view of Eqs. (1) and (4) the strength of the magnetic field $B_{\mathrm{e}}$ is then calculated using the formula:

$B_{\mathrm{e}}=k \Delta \lambda_{B}$.

An internal polarimeter possibility allows us to control the presence or absence of significant stochastic or spurious timedependent Stokes signatures. When using more than one pair of exposures, spectra with identical circular polarization, which are obtained at identical quarter-wave plate angles, are projected on the same location of the CCD. Therefore, we can calculate the value of the spurious "magnetic field" that must be equal to zero if all spurious effects are negligible.

Denote a center of gravity of a line with right circular polarization obtained during the first exposure by $\lambda_{m, r}$ and with right circular polarization obtained during the next exposure with identical quarter-wave plate angle by $\lambda_{m+1, r}$. The same designations for the left circular polarization are $\lambda_{m, l}$ and $\lambda_{m+1, l}$. Then

$B_{\text {test }}^{\prime}=k\left(\lambda_{m, r}-\lambda_{m+1, r}\right) / 2$

and

$B_{\text {test }}^{\prime \prime}=k\left(\lambda_{m, l}-\lambda_{m+1, l}\right) / 2$

for the other pair. As a result of this procedure, in view of Eqs. (1) and (4), the spurious "magnetic field" is

$B_{\text {test }}=k \Delta \lambda_{B} \equiv 0$

if all spurious effects are negligible (see also Donati et al. 1997; Wade et al. 2006). In order to test the reliability of obtained results, we evaluate the "spurious field" $B_{\text {test }} \pm \sigma_{\text {test }}$. To demonstrate that we have no systematic instrumental shift, we reproduce here the results of our investigations of stability of the instrumental zero point over the long time interval from 1989 to 2002. The systematic instrumental shift determined using 27 observational nights during $1989-1997$ is equal to $B_{\mathrm{e}}=-0.12 \pm 0.99 \mathrm{G}$ (Plachinda \& Tarasova 1999). In addition in this paper, we determined the systematic instrumental shift using $N=1491$ highaccuracy measurements of the magnetic field of four cool supergiants (Plachinda 2005) $-\alpha$ Aqr (G0Ib), $\beta$ Aqr (G2Ib), $\epsilon$ Gem (G8Ib), and $\epsilon$ Peg (G0Ib) - obtained during 23 observational nights from 1994 to 2002 . The mean value is equal to

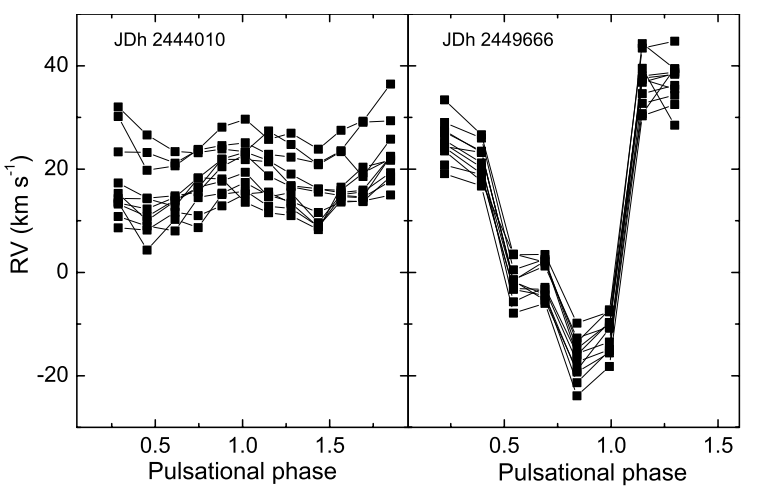

Fig. 1. Radial velocities measured in the UV lines Si III 1294.54, Si III 1296.73, Si III 1301.15, Si III 1303.32, Si III 1417.24 and in the UV doublets Si IV (1393.76, 1402.77), C IV (1548.20, 1550.77), and Al III $(1854.72,1862.79)$. The pulsation phases are calculated using the ephemeris given in Sect. 5.2, and the radial velocity of the system is $v_{\mathrm{r}}=2.77 \pm 0.02 \mathrm{~km} \mathrm{~s}^{-1}$ (see Sect. 4).

$B_{\mathrm{e}}=-0.44 \pm 0.38 \mathrm{G}$. Thus, we conclude that long-term systematic effects are absent within the observational error $<1 \mathrm{G}$.

In the case of our observations the coincidence between the numerical simulation value of the standard deviation (Monte-Carlo method) and the experimental standard deviation equals to $3.2 \%$ (Plachinda 2004, 2005). This discrepancy appears to be very small. Before each run of observations, the adjustment of the polarimeter was examined using a bright star. The adjustment of our stokesmeter is stable during long time interval from our first systematic observations in 1987 to the present time (Plachinda 2004, 2005).

\section{UV stellar wind variations}

Two sets (HJD 2444010 and 2449666) of the IUE observations of $\gamma$ Peg were longer than the 0.15-day pulsation period of the star. Figure 1 shows the variability of radial velocities measured in the cores of eleven individual spectral lines, including the wind-sensitive resonance doublets C IV, Si IV, and Al III. The pulsational variation of the UV velocity was present during both dates. Abnormal pulsation amplitude of about $60 \mathrm{~km} \mathrm{~s}^{-1}$ was detected in the second series. We were not able to decide whether the abnormal pulsation amplitude is real or caused by a problem in the IUE wavelength scale for a few spectra.

To eliminate the pulsation effect, the search for the long-term variability in the stellar wind was performed using the spectra obtained during the same pulsation phases (0.2-0.3): swp03946, swp05249, swp05256, swp52770, and swp52777.

The values of the expected variability, $\sigma_{\text {obs }} / \sigma_{\exp }$, were obtained using a statistics developed by Fullerton et al. (1996). A model of noise for the SWP-camera $S / N=A * \tanh (F / B)$ was used according to Henrichs et al. (1994), where $F$ is the flux, the maximum averaged signal to noise ratio $A=18$, and the average (normalized) flux level $B=1.009$.

The upper section of each panel in Fig. 2 shows an overplot of the CIV, Si IV, and Al III profiles along with the variability signatures in the lower sections. The ratio $\sigma_{\text {obs }} / \sigma_{\text {exp }}$ higher than unity indicates the level of variability, and the ratio $\sigma_{\mathrm{obs}} / \sigma_{\exp }$ close to unity indicates the magnitude of errors. The stellar wind variability is seen most clearly in the case of Al III (see the bottom panels of Fig. 2).

A specific type of the stellar wind variation demonstrates reliable signatures for the presence of a magnetic field on a star and enables the rotation period to be determined accurately, as in 

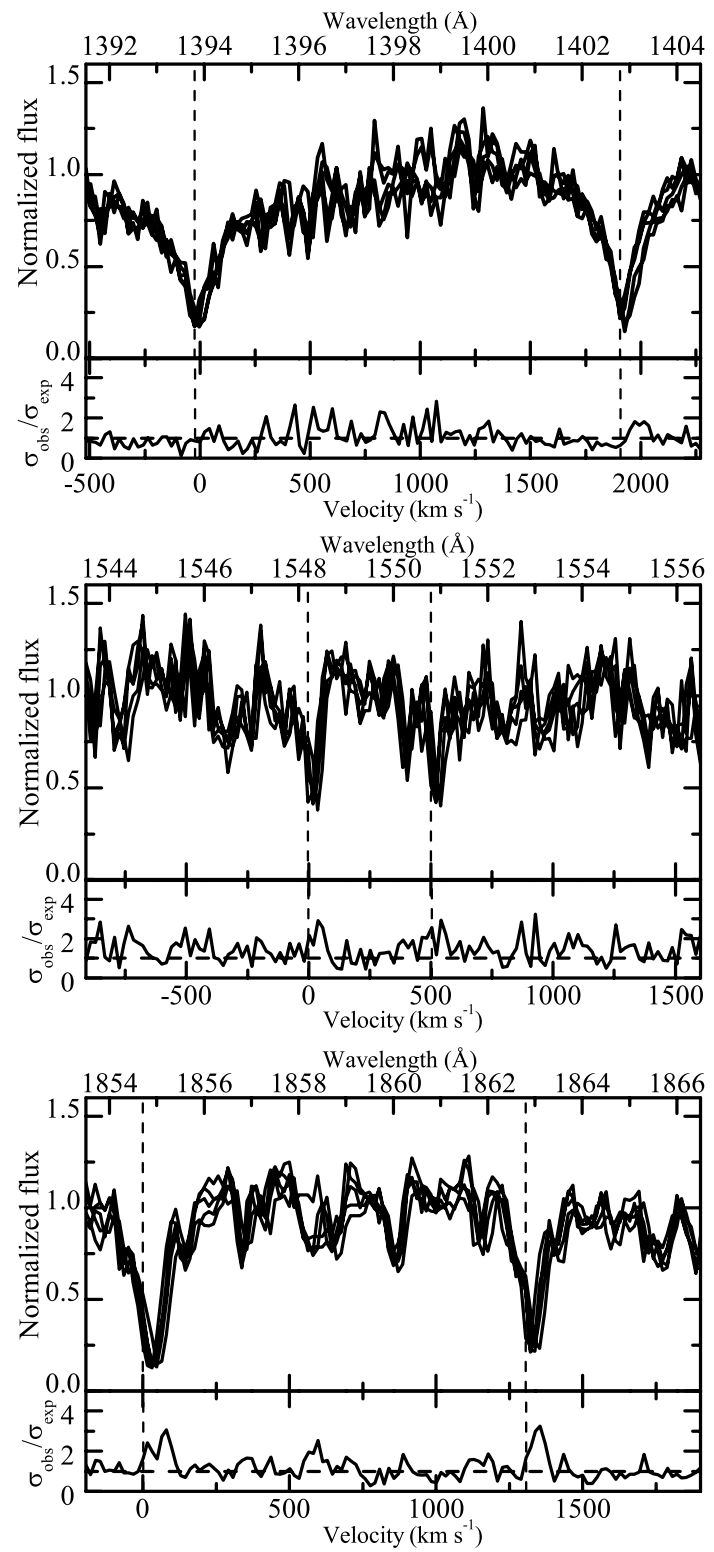

Fig. 2. Variation of the Si IV, C IV, and Al III (upper part of each panel) along with the variability signatures (bottom part of each panel). Fluxes are normalized to mean intensity of 1 .

cases of V 2052 Oph, $\zeta$ Cas, and $\omega$ Ori (Neiner et al. 2003a-c). However, in the case of $\gamma$ Peg, we cannot determine the rotation period from the variation of the stellar wind because of a small sample of the UV data. But Fig. 2 demonstrates that a variability is present in the stellar wind of $\gamma$ Peg.

\section{Binarity}

McNamara (1955) pointed out a possibility that $\gamma$-axis of the 0.15-day velocity curve of $\gamma$ Peg varies. Harmanec et al. (1979) determined the 6.83-day period for the variations in the $\gamma$-axis and concluded that the star is a spectroscopic binary with a circular orbit and low orbit inclination. Ducatel et al. (1981) also detected day-to-day variation in the $\gamma$-axis, but they supposed that the variation can be associated with stellar oscillations.

Our previous investigation of the possible origin in the 6.83-day variation of the $\gamma$-velocity of $\gamma$ Peg (Butkovskaya et al. 2006) led to the conclusion that the star is a spectroscopic
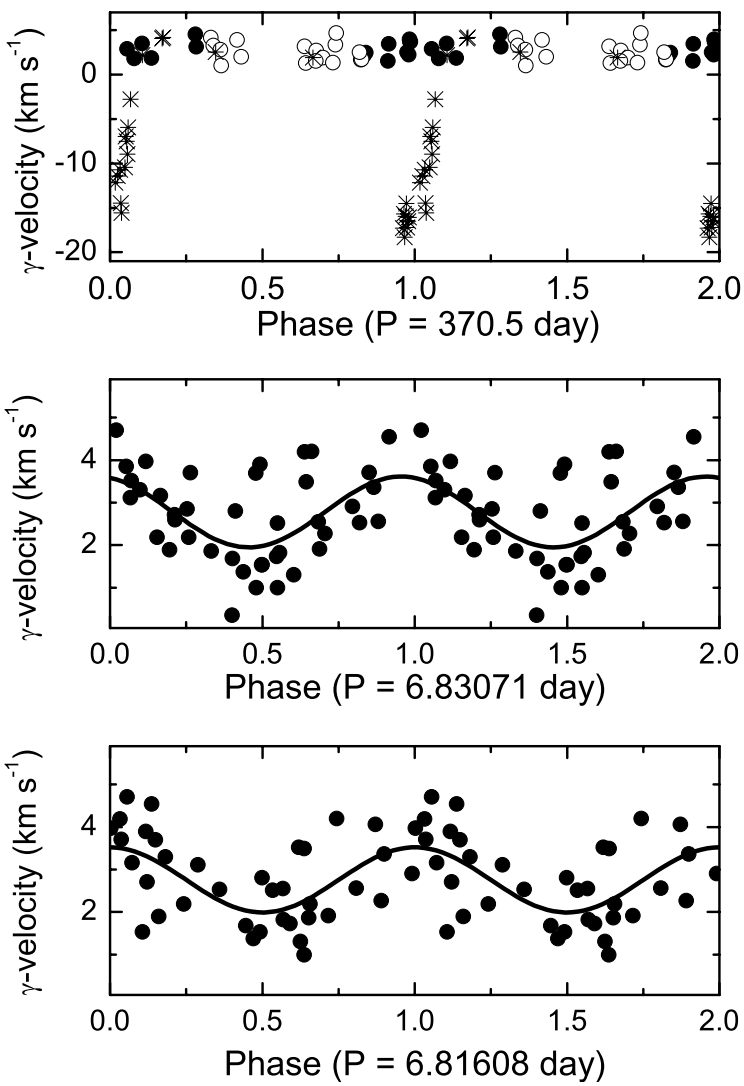

Fig. 3. Top panel: $\gamma$-velocities of McNamara (1955, 1956), Sandberg \& McNamara (1960), Lane \& Percy (1979), Ducatel et al. (1981) (open circles), Chapellier et al. (2006) (asterisks), and our data (filled circles) folded in phase with the 370-day period; middle panel and bottom panel: the positive $\gamma$-velocities folded in phase with 6.83071-day and 6.81608-day periods. The sinusoidal least-square fits are shown by solid lines.

binary as suggested by Harmanec et al. (1979). Recently, Chapellier et al. (2006) find another 370.5-day orbital period for this binary system. In this section we test the reality of the 370-day orbital period. We recalculated all $\gamma$-velocities and $2 K$-amplitudes of the individual pulsation curves using the least-square sinusoidal fitting of the data published by different authors. We found no statistically significant peculiarity in $2 K$-amplitude of the pulsation velocity curves, whereas the behavior of the $\gamma$-velocity is more complicated.

In the upper panel of Fig. 3, the $\gamma$-velocities obtained from data of McNamara (1955, 1956), Sandberg \& McNamara (1960), Lane \& Percy (1979), Ducatel et al. (1981), our data, and data of Chapellier et al. (2006) are folded in phase with the 370.5-day period.

Positive $\gamma$-velocities measured by us from 1998 to 2005 are located in the phase interval from 0.8 to 1.3. Extremely negative $\gamma$-velocities detected in 1992, 1993, and 1995 by Chapellier et al. (2006) occupy the same phase interval (0.9-1.1). It should be noted that during one observational night in 2005 (HJD 2453599), we also detected the abnormally blueshifted $\gamma$-velocity for the star of $-60.57 \pm 0.29 \mathrm{~km} \mathrm{~s}^{-1}$ (the value is located at phase 0.83 and is not shown in Fig. 3). In our opinion, these negative $\gamma$-velocities of the star repeatedly observed by Chapellier et al. (2006) and detected once by us are not produced by orbital motion. We believe that there is an episodic mass loss that takes place during sporadic outbursts by analogy with the situation for Be-stars. 
Table 2. Results of $\gamma$-velocity measurements of $\gamma$ Peg.

\begin{tabular}{rcccl}
\hline \hline No & HJD & $V_{\mathrm{r}}$ & rms & Authors \\
\hline 1 & 2434266.910 & 3.16 & 0.32 & McNamara $(1955,1956)$ \\
2 & 2434280.879 & 2.71 & 0.21 & $-/ /-$ \\
3 & 2434638.749 & 1.31 & 0.17 & \\
4 & 2434659.822 & 1.91 & 0.30 & \\
5 & 2434671.769 & 1.37 & 0.26 & \\
6 & 2434674.703 & 3.37 & 0.18 & \\
7 & 2434675.764 & 4.71 & 0.56 & \\
8 & 2434705.685 & 1.68 & 0.21 & \\
9 & 2434706.676 & 1.73 & 0.18 & \\
10 & 2436502.818 & 1.54 & 0.28 & Sandberg \& McNamara $(1960)$ \\
11 & 2439740.532 & 3.86 & 0.29 & Ducatel et al. $(1981)$ \\
12 & 2441189.807 & 4.16 & 0.16 & $-/ /-$ \\
13 & 2441192.786 & 3.31 & 0.12 & \\
14 & 2441201.762 & 2.79 & 0.19 & \\
15 & 2441202.708 & 1.00 & 0.50 & \\
16 & 2442645.827 & 1.53 & 0.19 & Lane \& Percy $(1979)$ \\
17 & 2448538.403 & 3.69 & 0.09 & Chapellier et al. $(2006)$ \\
18 & 2448539.500 & 4.19 & 0.04 & $-/ /-$ \\
19 & 2448884.556 & 2.19 & 0.08 & \\
20 & 2451060.481 & 2.27 & 0.22 & Present paper \\
21 & 2451061.480 & 3.71 & 0.26 & $-/ /-$ \\
22 & 2451088.430 & 2.91 & 0.25 & \\
23 & 2451171.218 & 4.54 & 0.13 & \\
24 & 2451172.251 & 3.11 & 0.27 & \\
25 & 2451488.272 & 1.86 & 0.32 & \\
26 & 2452217.366 & 3.52 & 0.30 & \\
27 & 2453280.317 & 2.55 & 0.23 & \\
28 & 2453283.283 & 3.97 & 0.17 & \\
29 & 2453284.284 & 3.70 & 0.19 & \\
30 & 2453418.277 & 2.56 & 0.70 & Chapellier et al. (2006) \\
31 & 2453536.550 & 1.90 & 0.08 & $-/ /-$ \\
32 & 2453598.455 & 2.19 & 0.29 & Present paper \\
33 & 2453600.448 & 2.52 & 0.18 & $-/ /-$ \\
34 & 2453627.426 & 1.54 & 0.18 & \\
35 & 2453628.421 & 3.49 & 0.08 & \\
36 & 2453689.294 & 1.82 & 0.29 & \\
& & & & \\
& &
\end{tabular}

All positive $\gamma$-velocities (in $\mathrm{km} \mathrm{s}^{-1}$ ) are listed in Table 2. In the middle panel of Fig. 3, all these $\gamma$-velocities are folded with the 6.83-day period, as suggested by Harmanec et al. (1979). One can see that some values demonstrate significant scatter. Therefore, to improve the orbital period we carefully re-analyzed the $\gamma$-velocity data presented in Table 2 using the "Period04" program of Lenz et al. (2005). On the basis of this analysis, the most probable period has been evaluated for which we adopt the ephemeris:

$\operatorname{HJD}\left(R V_{\max }\right)=2434266.421+n \times 6.81608 \pm 0.00012$.

The radial velocity of the system is $v_{\mathrm{r}}=2.77 \pm 0.02 \mathrm{~km} \mathrm{~s}^{-1}$.

In the bottom panel of Fig. 3, all $\gamma$-velocities are folded in phase with this corrected period. In spite of the small $\gamma$-velocities errors, one can see a significant scatter of data. To improve the orbital period and orbital elements, additional time-series of the high-accuracy radial velocity measurements are desirable.

Frolov et al. (1980) discuss the existence of the unusual "synchronization" between the pulsational and orbital motions in some close eclipsing and spectroscopic binaries containing pulsating components of different types. They found particularly, that Harmanec's orbital period of $\gamma \mathrm{Peg}, P_{\mathrm{orb}}=$ 6.830713 days, contains almost an integer number of pulsation cycles, $P_{\text {orb }} / P_{\text {puls }}=45.01$. They proposed a parameter $\Delta=\left|P_{\text {orb }} / P_{\text {puls }}-A\right|$ (where $A$ is the nearest integer) as the measure of deviation rate from the exact synchronization. In the
Table 3. Results of the magnetic field measurements.

\begin{tabular}{rccrrr}
\hline \hline No & HJD & Phase & $B_{\mathrm{e}}$ & $\sigma$ & $N$ \\
\hline 1 & 2450681.516 & 0.323 & -7.7 & 5.6 & 5 \\
2 & 2450684.495 & 0.771 & -2.6 & 3.6 & 24 \\
3 & 2450802.318 & 0.479 & -6.5 & 9.1 & 7 \\
4 & 2451060.481 & 0.278 & -5.0 & 6.3 & 26 \\
5 & 2451061.480 & 0.428 & 10.5 & 4.8 & 37 \\
6 & 2451088.430 & 0.479 & 0.8 & 6.0 & 25 \\
7 & 2451115.249 & 0.509 & -12.7 & 9.8 & 9 \\
8 & 2451487.362 & 0.434 & -13.9 & 15.0 & 4 \\
9 & 2451488.272 & 0.571 & -2.7 & 4.1 & 26 \\
10 & 2453280.317 & 0.897 & 45.1 & 12.7 & 5 \\
11 & 2453366.212 & 0.807 & 0.4 & 14.7 & 4 \\
12 & 2453598.455 & 0.710 & 6.4 & 7.8 & 13 \\
13 & 2453599.455 & 0.861 & 35.5 & 9.6 & 12 \\
14 & 2453600.448 & 0.010 & 46.2 & 10.9 & 9 \\
15 & 2453627.426 & 0.064 & 23.0 & 8.5 & 17 \\
16 & 2453628.421 & 0.214 & 2.8 & 9.8 & 13 \\
17 & 2453689.294 & 0.363 & 3.0 & 6.0 & 9 \\
\hline
\end{tabular}

case of $P_{\text {orb }}=6.830713$ day $\Delta=0.01$ and in the case of new $P_{\text {orb }}=6.81608$ day, the ratio of $P_{\text {orb }} / P_{\text {puls }}=44.92$ and $\Delta=0.08$.

Two suppositions concerning this "synchronization" have been proposed by the authors. The first one is that this may be an effect of the tidal action of the close companion on the pulsational process that accords with the well-known Fitch's hypothesis ("tidal deformations induced in the hydrogen and/or helium ionization zones of each primary by a faint companion, resulting in surface-zonal variations of the amplitude and phase of each primary's normal radial pulsations", Fitch 1967). The second hypothesis is that the "synchronization" is the result of the coincidence of an integer number of the pulsation periods and the axial rotation period of the same pulsating star. In that case, in particular, we expect the rotational period of $\gamma$ Peg to be close to the orbital period.

\section{Magnetic field}

The results of the magnetic field measurements are listed in Table 3. The Heliocentric Julian dates are given in the second column. The third column contains the phases of the period $6.6538 \pm 0.0016$ day (see below). The next three columns give the observed longitudinal magnetic field $B_{\mathrm{e}}$ averaged per night, the error bar $\sigma$, and the number of individual measurements $N$ that were used to estimate $B_{\mathrm{e}}$.

\subsection{Axial rotation}

We used our averaged per night measurements of the longitudinal magnetic field to search for a periodicity in the field strength variation. To determine the axial rotation period, we applied the "Period04" program. Computations were performed on the assumption that the rotation period of $\gamma$ Peg is close to its orbital period. Analysis of the power spectrum revealed the small family of frequencies, each of them could correspond to the rotation period. Due to an additional analysis, we conclude the period of 6.6538 days is the most probable rotation period, but additional observations are needed in order to confirm this result. We adopt the ephemeris:

$\operatorname{HJD}\left(B_{\max }\right)=2450679.364+n \times 6.6538 \pm 0.0016$.

The period obtained in this manner is close to the orbital one, but a small disagreement between the periods exists. 


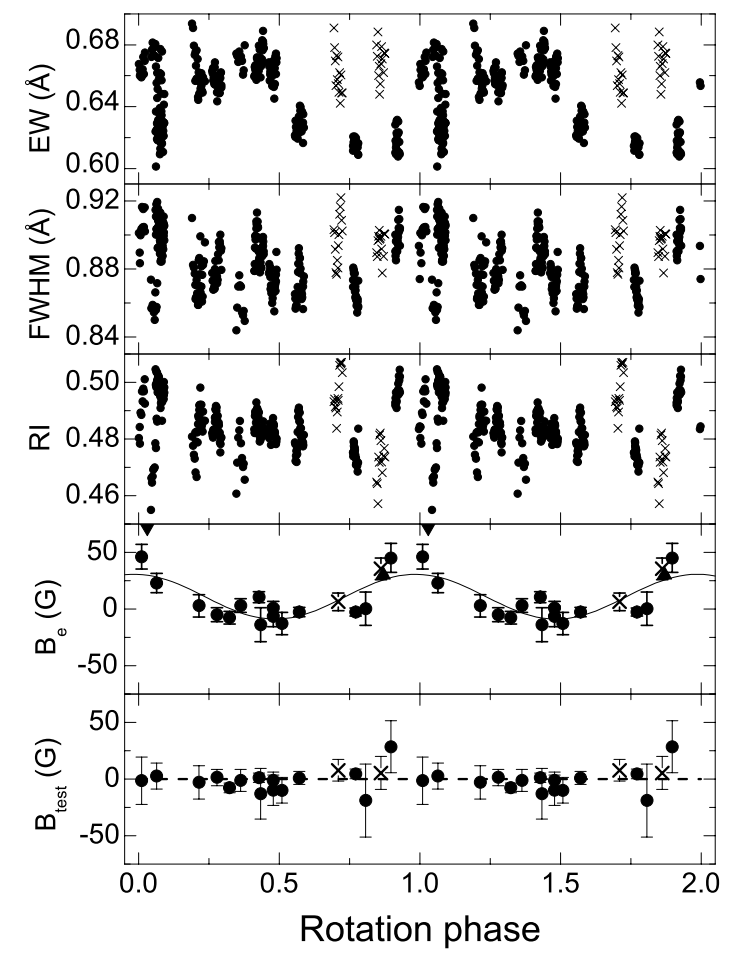

Fig. 4. $E W, F W H M$, RI, $B_{\mathrm{e}}$, and zero field $B_{\text {test }}$ folded in phase with the 6.6538-day axial rotation period. The line parameters and magnetic fields, obtained during two nights of observations, HJD 2453599 (when $\gamma$-velocity of the star was unusually blueshifted, see Sect. 4), and the previous night HJD 2453598 (when the $\gamma$-velocity was normal but the increase in the $E W$ was detected), are plotted as crosses. Our other data are shown as filled circles. Two more precise magnetic field values, obtained by Rudy \& Kemp and Landstreet are shown as filled up- and down-directed triangles. The best sinusoidal fit of the magnetic data is presented by the solid line.

Tan Huisong et al. (1993) propose using parameter $S_{\text {orb }}=$ $\left|\left(P_{\text {orb }}-P_{\text {rot }}\right) / P_{\text {orb }}\right|$ to determine the synchronism in binary systems. According to their criterion, the synchronism should be considered as established if $S<0.01$. If $S>0.04$ the synchronism is absent in a system. The case for which $0.01<S<0.04$ indicates an intermediate situation when the system demonstrates slight asynchronism or significant differential rotation. In the case of $\gamma$ Peg, $S=0.024$.

We find for $\gamma$ Peg that both the orbital and the rotational periods are integral multiples of the difference between them:

$$
\begin{gathered}
1 / S_{\text {orb }}=P_{\text {orb }} /\left|P_{\text {orb }}-P_{\text {rot }}\right|=42.002, \\
1 / S_{\text {rot }}=P_{\text {rot }} /\left|P_{\text {orb }}-P_{\text {rot }}\right|=41.002 .
\end{gathered}
$$

In the case of $P_{\text {orb }}=6.830713$ day, $1 / S_{\text {orb }}=38.611 \neq$ Integer .

In Fig. 4 the equivalent width $(E W)$, full width at half maximum $(F W H M)$, and residual intensity (RI) of the He I line, as well as the mean-per-night longitudinal magnetic field $B_{\mathrm{e}}$ and zero field $B_{\text {test }}$ are folded in phase with the axial rotation period. Zero-field $B_{\text {test }}$ measurements (see Fig. 4 ) indicate that no spurious circular polarization signals are observed.

The EWs of the He I line exhibit two maxima at phases 0.05 and 0.45 (i.e. near the phases of maximal positive and negative field, respectively) and two minima at phases 0.25 and 0.75 . The same behavior of the FWHM and RI can be suspected. Variations in the RI of optical photospheric lines with rotational phase have also been observed in V $2052 \mathrm{Oph}$ and $\zeta$ Cas (Neiner et al. $2003 a, c)$. The authors explain these variations by a temperature difference between the magnetic poles and the rest of the stellar surface. Assuming a model of an oblique magnetic rotator, they suggest that temperature variations occur when the magnetic poles pass through the line of sight. The suggested model explains the observed rotational variability of the spectral lines.

In total, 9 measurements of the magnetic field on $\gamma$ Peg were also performed by Rudy \& Kemp (1978), and 3 measurements were made by Landstreet (1982). In spite of large errors, two more precise values obtained by Rudy \& Kemp (30 $\pm 90 \mathrm{G})$ and Landstreet $(72 \pm 45 \mathrm{G})$ agree well with our data. We do not illustrate the other results of these authors due to unacceptable error bars (more than one hundred Gauss).

We find the longitudinal magnetic field varies from $-10 \mathrm{G}$ to $+30 \mathrm{G}$, the average value $B_{0}=11 \pm 3 \mathrm{G}$, and the amplitude $B_{\mathrm{e}}=20 \pm 4$ G. Using the Fisher's F-test, a confidence level of a statistical assurance of the field variation with rotation is evaluated as $96 \%$. Assuming the limb darkening coefficient $u=0.350$ we simulated the dipole magnetic field of $\gamma$ Peg using the "magnetic charge distribution" method presented and described by Lebedev (1980) and Gerth \& Glagolevskij (2000). The best fit to the observational data in the case of the centered dipole gives the following results: the polar field strength $B_{\text {pol }}=570 \mathrm{G}$, the angle between spin axis and line of sight $i=9^{\circ}$, and the angle between both the spin and dipole axes $\beta=85^{\circ}$. We do not show the fit in Fig. 4 because it differs only slightly from the sinusoidal fit obtained by least-square method. In view of the aforesaid, the centered dipole is a good initial approximation to describe the magnetic field variability due to axial rotation of $\gamma$ Peg.

\subsection{Pulsations}

A study of the magnetic-field behavior due to the radial pulsations of the star has been performed using individual longitudinal magnetic field values. The mean per-night magnetic fields were subtracted from the individual values to eliminate the axial rotation effect. Therefore, we cannot claim that the effective magnetic field of $\gamma$ Peg changes its sign during the pulsation. The phases of the 0.15-day pulsation period were computed according to the ephemeris given by Butkovskaya \& Plachinda (2004):

$$
\begin{aligned}
\operatorname{HJD}\left(R V_{\max }\right)= & 2451060.461+n \times 0.151750393 \\
& \pm 6.4 \times 10^{-8}
\end{aligned}
$$

In the upper panel of Fig. 5, the acceleration and radius variation calculated in a standard manner are presented with the radial velocity in the stellar rest frame. The curves are normalized by their extreme values, which are $1.41 \mathrm{~m} \mathrm{~s}^{-2}$ for the acceleration, $2.98 \mathrm{~km} \mathrm{~s}^{-1}$ for velocity, and $0.004 R_{*}$ for the peak-to-peak amplitude of the radius variation. The smooth sinusoidal form of all these curves indicates the absence of significant non-linear motions in the photosphere layer where the He I line is formed.

The longitudinal magnetic field phased with the pulsation period is represented in the second panel of Fig. 5. The $B_{\mathrm{e}}$ shows periodic variations due to the stellar radial pulsation. The behavior of the magnetic field with the pulsation period can be seen more clearly for averaged data. Therefore the data are binned and averaged within 7 bins in the third panel of Fig. 5. Each bin consists of about 30 field measurements. The magnetic field varies with the amplitude of $B_{\mathrm{e}}=7.2 \pm 0.6 \mathrm{G}$ around the average value of $B_{0}=0.5 \pm 0.4 \mathrm{G}$.

To test the reliability of the pulsational variation of the magnetic field, "zero" field $B_{\text {test }}$ are also binned and averaged within 7 bins and phased with the same pulsation period in the bottom panel. The average value is $\left\langle B_{\text {test }}\right\rangle=3.86 \pm 2.07 \mathrm{G}$. 


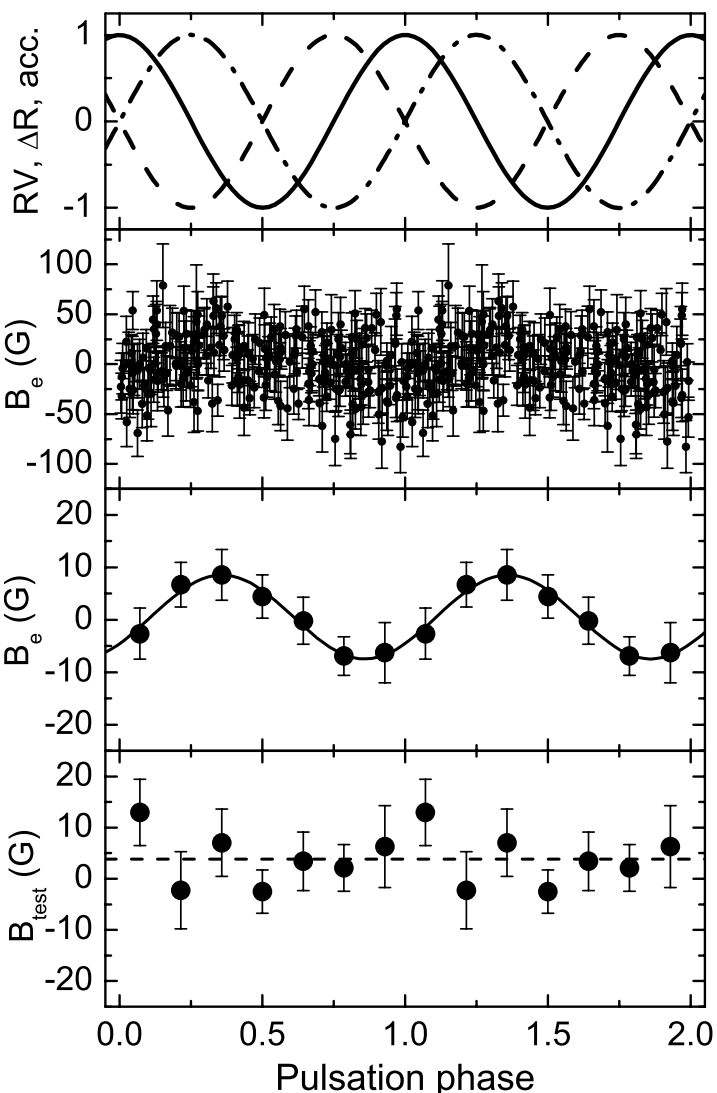

Fig. 5. Top panel: radial velocity curve (solid line), acceleration curve (dash-dotted line), and radius variation curve (dashed line); middle panel: individual magnetic field values folded in phase with the 0.15-day pulsation period; two bottom panels: magnetic field $B_{\mathrm{e}}$ and "zero" magnetic field $B_{\text {test }}$ binned at 7 bins; the least-square sinusoidal fit is shown by the solid line.

What is the origin of the observed pulsational variations of the longitudinal magnetic field?

1. Owing to the magnetic field being frozen into the plasma, the initial hypothesis is that the radial pulsations of the star result in the homothetic variation of the magnetic dipole. Numerical simulations were performed to evaluate the amplitude of the longitudinal component variation in this case. For a given configuration of the dipole $\left(B_{\mathrm{pol}}=570 \mathrm{G}, i=9^{\circ}\right.$, $\beta=85^{\circ}$ ), the observed amplitude of the longitudinal magnetic field variation must be some tenths of Gauss, which is less than the measured amplitude by an order of magnitude.

2. In reality, the variation of the stellar structure during a pulsation cycle is not homologous. As reviewed by Osaki (1993), the excitation mechanism of the pulsations in the $\beta$ Ceptype stars is a well-known $\kappa$ mechanism, but due to the heavy element opacity bump at the "transition zone" around $T \approx 2 \times 10^{5} \mathrm{~K}$. The pulsational amplitude of the layers above the "transition zone" is significantly larger than the pulsational amplitude of the layers beneath this "transition zone". Therefore, while under the "transition zone" the magnetic field geometry is virtually stable, the pulsational motion of the atmosphere matter can appreciably distort the initial configuration of the magnetic dipole.

3. In the presence of a magnetic field, the pulsational motion of upper layers of a star may lead to generation of magnetohydrodynamic waves. In that case the initial shape and density of magnetic field lines would also be disturbed.

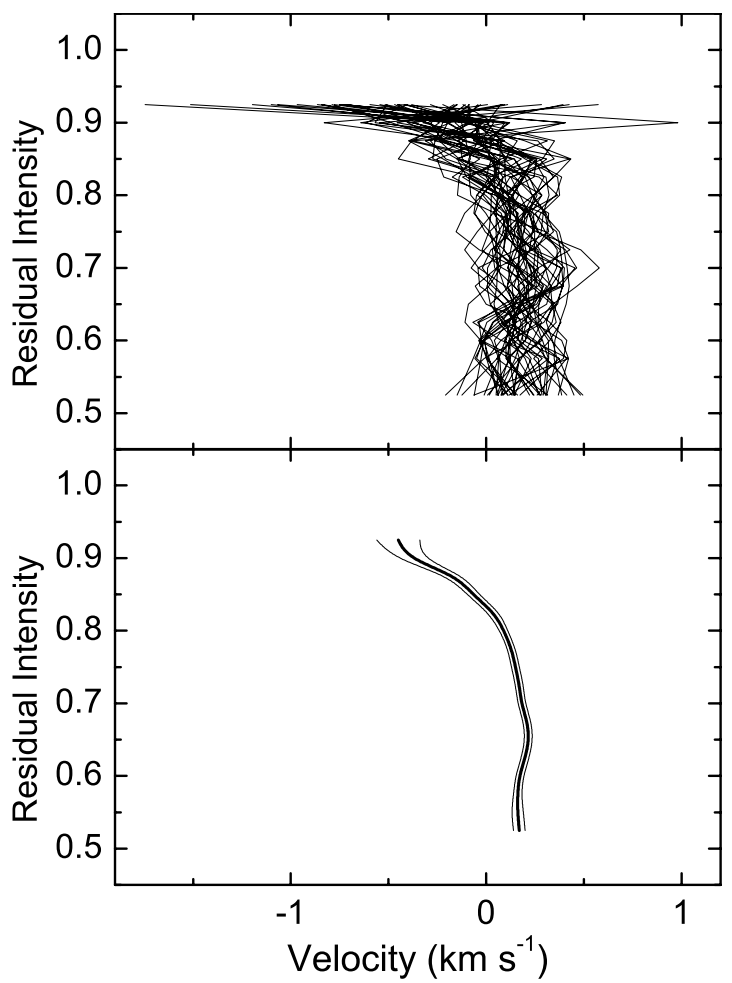

Fig. 6. Top panel: bisectors of the He I 6678 line obtained during $4.93 \mathrm{~h}$ (47 exposures; HJD 2451172); bottom panel: mean bisector (thick line) and RMS (thin lines on either side of the mean). The zero point of velocity scale corresponds to a center of gravity of the line.

4. In addition, the detected pulsational variations of the longitudinal magnetic field may be an artificial effect produced by a variable velocity field. A possible signature of the velocity field is the presence of line asymmetries, usually described in terms of the shape of the line bisector. Figure 6 shows the asymmetries for He I 6678, as delineated by the line bisectors, with individual exposures in the top panel and their mean in the bottom one.

All the above-mentioned mechanisms are probably capable of giving the pulsational variation of the longitudinal magnetic field. Therefore magnetohydrodynamic simulations are needed for studying the relation between stellar pulsations and magnetic field behavior. In addition, numerical simulations of the synthetic spectra of a star in the presence of a magnetic field and variable velocity field are also needed.

\section{Summary}

We have presented new spectropolarimetric study of the classical $\beta$ Cep-type star $\gamma$ Peg.

- It is shown that the UV stellar wind exhibits variability. The pulsational variations of the wind velocity were detected during two nights. We do not have enough data to distinguish whether the abnormal pulsation amplitude found for the second night is real or caused by a problem with the IUE radial velocities.

- We do not confirm the 370.5-day orbital period, but do conclude that the star is a spectroscopic binary with probable orbital period $P_{\text {orb }}=6.81608 \pm 0.00012$ day. The radial velocity of the system is $v_{\mathrm{r}}=2.77 \pm 0.02 \mathrm{~km} \mathrm{~s}^{-1}$. 
- The abnormally blueshifted radial velocities were detected in the He I 6678 line $\left(\gamma=-60.57 \pm 0.29 \mathrm{~km} \mathrm{~s}^{-1}\right)$ during a single night.

- The weak magnetic field has been detected on $\gamma$ Peg. The longitudinal component of the field varies from $-10 \mathrm{G}$ to $30 \mathrm{G}$ in phase with the stellar rotation. The most probable rotation period is $P_{\text {rot }}=6.6538 \pm 0.0016$ day, but additional data, which will require further observations, are needed in order to confirm these results.

- We find that both the orbital and the rotational periods are integral multiples of the difference between them: $P_{\text {orb }} / \mid P_{\text {orb }}-$ $P_{\text {rot }} \mid=42.002$, and $P_{\text {rot }} /\left|P_{\text {orb }}-P_{\text {rot }}\right|=41.002$.

- Assuming the magnetic field of $\gamma$ Peg as a dipole, we have estimated the polar field strength $B_{\mathrm{pol}}=570 \mathrm{G}$. The angle between the rotational axis and line of sight $i=9^{\circ}$. The angle between the rotational and magnetic dipole axes $\beta=85^{\circ}$.

- The longitudinal component of the magnetic field was found to be variable during the 0.15 -day pulsation period of the star with the amplitude $\sim 7 \mathrm{G}$.

- Qualitative analysis of the origin of the observed pulsational variations of the longitudinal magnetic field led to the conclusion that magnetohydrodynamic simulations are needed for studying the relation between stellar pulsations and magnetic field behavior. Numerical simulations of the synthetic spectra of a star in the presence of both the magnetic field and variable velocity field are also needed.

Acknowledgements. The authors wish to thank P. Harmanec, G. Valyavin, and W. Weiss for their insightful comments and useful suggestions. We thank the referee, J. Landstreet, for a careful review of the paper resulting in insightful recommendations that strengthened the paper. We thank the language editor, Joli Adams, for the kind suggestions on improving of the paper. SIP acknowledges support in part from the Austrian Science Funds (P17890).

\section{References}

Babcock, H. W. 1958, ApJS, 3, 141

Borra, E. F., Fletcher, J. M., \& Poeckert, R. 1981, ApJ, 247, 569

Butkovskaya, V. V., \& Plachinda, S. I. 2004, JQSRT, 88, 17

Butkovskaya, V., Khan, S., Plachinda, S., Rostopchin, S., \& Shulyak, D. 2006, Izv. Krym. Astrofiz. Obs., 103, in press

Chadid, M., Wade, G. A., Shorlin, S. L. S., \& Landstreet, J. D. 2004, A\&A, 413, 1087

Chapellier, E., Le Contel, D., Le Contel, J. M., et al. 2006, A\&A, 448, 697
Charbonneau, P., \& MacGregor, K. 2001, ApJ, 559, 1094

de Jager, C., Sato, N., Burger, M., \& Neven, L. 1982, ApSS, 83, 411

Donati, J.-F., Semel, M., Carter, B. D., Rees, D. E., \& Collier Cameron, A. 1997, MNRAS, 291, 658

Donati, J.-F., Wade, G. A., Babel, J., et al. 2001, MNRAS, 326, 1265

Donati, J.-F., Babel, J., Harries, T. J., et al. 2002, MNRAS, 333, 55

Donati, J.-F., Howarth, I. D., Jardine, M. M., et al. 2006, MNRAS, 370, 629

Ducatel, D., Le Contel, J.-M., Sareyan, J.-P., \& Valtier, J.-C. 1981, A\&AS, 43, 359

Fitch, W. S. 1967, ApJ, 148, 481

Frolov, M. S., Pastukhova, E. N., Mironov, A. V., \& Moshkalev, V. G. 1980, IBVS, 1894

Fullerton, A. W., Gies, D. R., \& Bolton, C. T. 1996, ApJSS, 103, 475

Gerth, E., \& Glagolevskij, Yu. V. 2000, In Proceedings of the International Meeting Magnetic fields of chemically peculiar and related stars, ed. Yu. V. Glagolevskij, \& I. I. Romanyuk

Harmanec, P., Koubsky, P., Krpata, J., \& Zdarsky, F. 1979, IBVS, 1590, 1

Henrichs, H. F., Kaper, L., \& Nichols, J. S. 1994, A\&A, 285, 565

Hubrig, S., Briquet, M., Scholler, M., et al. 2006, MNRAS, 369, L61

Lane, M. C., \& Percy, J. R. 1979, AJ, 84, 831

Landstreet, J. D. 1982, ApJ, 258, 639

Lebedev, V. S. 1980, Astrof. Issled., 12, 25

Lenz, P., \& Breger, M. 2005, CoAst, 146, 53

McNamara, D. H. 1953, PASP, 65, 144

McNamara, D. H. 1955, ApJ, 122, 95

MacDonald, J., \& Mullan, D. J. 2004, MNRAS, 348, 702

Moss, D. 1989, MNRAS, 236, 629

Mullan, D. J., \& MacDonald, J. 2005, MNRAS, 356, 1139

Neiner, C., Geers, V. C., Henrichs, H. F., et al. 2003a, A\&A, 406, 1019

Neiner, C., Hubert, A.-M., Fremat, Y., et al. 2003b, A\&A, 409, 275

Neiner, C., Henrichs, H. F., Floquet, M., et al. 2003c, A\&A, 411, 565

Osaki, Y. 1993, ASP Conf. Ser., 40, 512

Plachinda, S. I. 2000, A\&A, 360, 642

Plachinda, S. I. 2004, in Photopolarimetry in Remote Sensing, ed. G. Videen, Ya. S. Yatskiv, \& M. I. Mishchenko (Kluwer Acad. Publ.), 351

Plachinda, S. I. 2005, Astrophysics, 48, 9

Plachinda, S. I., \& Tarasova, T. N. 1999, ApJ, 514, 402

Preston, G. W. 1967, The Magnetic and Related Stars, ed. R. C. Cameron, Mono Book Corporation, Baltimore, 26

Romanov, Yu. S., Udovichenko, S. N., \& Frolov, M. S. 1987, AZh Lett., 13, 69

Romanov, Yu. S., Udovichenko, S. N., \& Frolov, M. S. 1994, Bul. Spec. Astrophys. Obs., 38, 169

Rudy, R. J., \& Kemp, J. C. 1978, MNRAS, 183, 595

Sandberg, H. E., \& McNamara, D. H. 1960, PASP, 72, 508

Smith, M. A., \& McCall, M. L. 1978, ApJ, 221, 861

Spruit, H. C. 2002, A\&A, 381, 923

Tan, H., Wang, X., Pan, K., et al. 1993, ASP Conf. Ser., 38, 374

Wade, G. A., Chadid, M., Shorlin, S. L. S., Bagnulo, S., \& Weiss, W. W. 2002, A\&A, 393, L17

Wade, G. A., Fullerton, A. W., Donati, J.-F., et al. 2006, A\&A, 451, 195 